\title{
Nanoflower-based SERS active particles for chemosensing
}

\author{
Susanne Pahlow ${ }^{1}$ Dana Cialla ${ }^{1,2}$, Anne März ${ }^{1}$, Karina Weber ${ }^{1,2}$, Jürgen Popp ${ }^{1,2}$ \\ ${ }^{1}$ Friedrich-Schiller University Jena, Institute of Physical Chemistry and Abbe Center of Photonics, \\ Helmholtzweg 4, 07745 Jena, Germany, \\ ${ }^{2}$ Institute of Photonic Technology, Albert-Einstein-Straße 9, 07745 Jena, Germany \\ juergen.popp@uni-jena.de
}

\begin{abstract}
:
A new facile method for the modification of magnetic beads with a SERS active substrate is introduced. The technique involves enzymatically induced silver deposition. Horse radish peroxidase is immobilized on streptavidin modified beads using a short biotinylated oligonucleotide as linker molecule. The enzyme induces the reduction of the silver ions to elementary silver, resulting in the growth of flower-like silver nanoparticles on the surface of the magnetic beads. Due to the unique structure of the silver nanoflowers they provide an enhancement of the inherently weak Raman signal of analyte molecules. In this study the structure of the silver modified magnetic beads and their SERS properties are investigated using adenine as model analyte.
\end{abstract}

Key words: surface enhanced Raman spectroscopy (SERS), silver nanoflowers, magnetic beads, adenine

\section{Introduction}

Nanostructured metal surfaces are essential for the application of surface enhanced Raman spectroscopy (SERS) as this technique is based on inducing an enhanced electromagnetic field at metal nanostructures [1-4]. Molecules close to the metal surface are affected by the enhanced field resulting in a significantly enhanced Raman signal intensity of these molecules. In order to achieve such an enhanced electromagnetic field localized surface plasmon polaritions have to be excited. Therefore, the wavelength of the incident laser light has to overlap with the plasmonic absorption profile of the nanostructured metal surface. Common metal nanostructures applied as SERS active substrate are silver and gold colloidal solutions [5-7], nanostructures produced by lithographic techniques [8-11] or rough metal electrodes [12,13]. Most of these applied SERS active substrates provide a narrow absorption profile and are designed for precise applications in specific wavelength regions. SERS substrates known to provide a rather broad plasmonic absorption profile are enzymatically generated silver nanoparticles (EGNP) [14]. These nanoparticles exhibit a nanoflower-like structure which is well suited for the application as SERS active substrates [15]. EGNP can be produced very easily and cost efficient and are applicable with excitation wavelength within the whole visible range [14]. The potential of these substrates for SERS measurements has already been shown successfully by Strelau and co-workers $[14,15]$.

The application of SERS as powerful analytical detection method is becoming more and more of interest especially in the field of bioanalytics and medical diagnostics [16-23]. This is due to the high sensitivity and fingerprint specificity of SERS. For establishing SERS as routine detection method in this application field suitable SERS substrates have to be provided. However, investigation of bioanalytical and

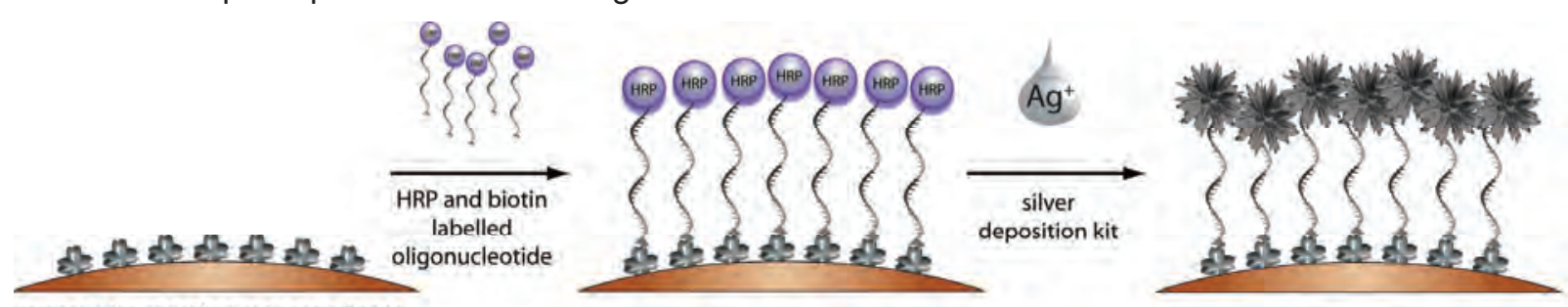

streptavidin modified magnetic beads

Fig. 1. Scheme of the preparation of silver nanoflowers on magnetic beads 
medical samples still remains a major challenge due to often inevitable sample preparation. The molecules of interest are mostly located in complex matrices as e.g. blood and urine. For this reason research is focused on the development of particles, which provide beside the local field enhancement, properties applicable for sample preparation steps [24,25]. A novel approach is the preparation of magnetic particles covered with SERS active metal. The magnetic properties can be used for sample preparation, enrichment and separation steps as well as for localizing the particles for detection [26-32]. So far magnetic particles covered with gold and silver have been introduced applying chemical reduction mechanisms.

In this contribution a new approach for modifying magnetic particles with a SERS active substrate is presented. Magnetic beads were covered with enzymatically generated nanoflowers combining a unique silver surface structure suited for SERS detection with magnetic properties applicable to manipulate the particles for preparation and detection procedures. These SERS active magnetic beads were tested concerning their performance for SERS measurements.

\section{Experimental details}

For the preparation of silver nanoflowers on magnetic beads, streptavidin-modified magnetic beads were purchased from PerkinElmer Chemagen Technologie $\mathrm{GmbH}$ (Baeseweiler, Germany) with an initial concentration of $25 \mathrm{mg} / \mathrm{ml}$. $10 \mu \mathrm{l}$ of these magnetic beads were washed twice with $150 \mu$ storage buffer (1xPBS - phosphate buffered saline $0.1 \%$ BSA - bovine serum albumin) and resuspended in $148.5 \mu \mathrm{l}$ storage buffer. Consequently $1.5 \mu \mathrm{l}$ of $100 \mu \mathrm{M}$ horseradish peroxidase (HRP) and biotin labelled oligonucleotide were added and the solution was incubated for $30 \mathrm{~min}$ at room temperature with slow tilt rotation. Due to the interaction of streptavidin and biotin, the oligonucleotides were bound to the surface of the magnetic beads. Subsequently the beads were washed three times with $150 \mu$ l storage buffer, twice with distilled water and resuspended in $50 \mu \mathrm{l}$ distilled water. Applying $455 \mu \mathrm{l}$ of the EnzMet reagent from Nanoprobes (Yaphank, NY, USA) the silver deposition in nanoflower-like structures takes place based on an enzymatically (HRP) driven mechanism. After $5 \mathrm{~min}$ the particles were washed twice with distilled water, once with citrate buffer and finally resuspended in $50 \mu \mathrm{l}$ citrate buffer for
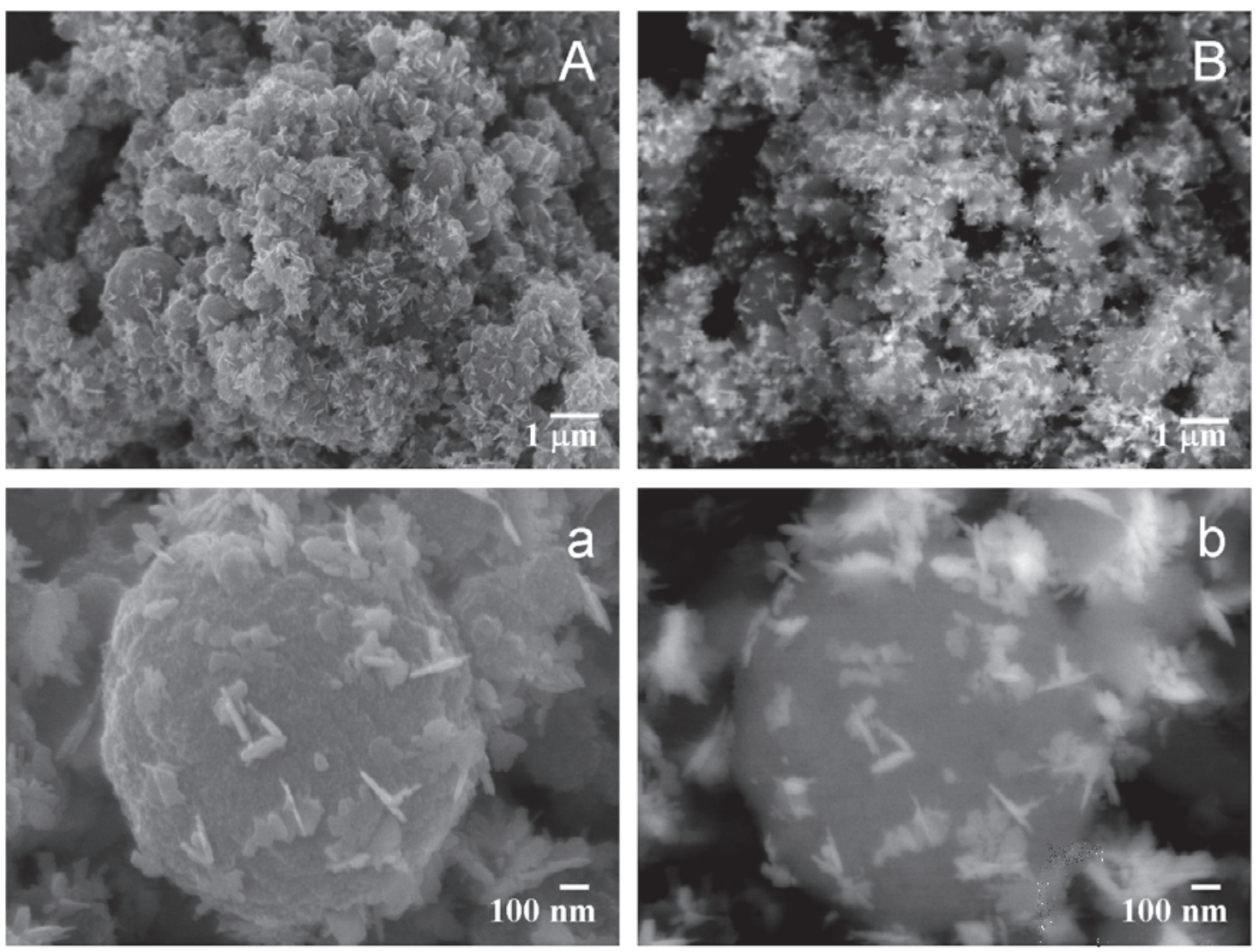

Fig. 2. SEM ( $A$ and a) and contrast images ( $B$ and $b$ ) of magnetic beads covered with enzymatically generated silver nanoflowers 
storage. Before the SERS measurements, the particles were washed again with distilled water three times and resuspended in $200 \mu$ l distilled water. In Figure 1, a theoretical scheme for the preparation of silver nanoflowers on magnetic beads is shown. Figure 2 presents scanning electron microscopy (SEM) images ( $A$ and $a$ ) and SEM images in backscatter electron imaging mode ( $B$ and $b$ ) of magnetic beads covered with enzymatically generated silver nanoflowers (see detailed explanation in section 'results and discussion').

For the SERS measurements adenine of analytical grade was purchased at Sigma Aldrich and dissolved in distilled water to prepare the dilutions. The SERS measurements were carried out at a conventional microRaman setup (LabRam, Horiba Jobin-Yvon) equipped with a 300 lines $/ \mathrm{mm}$ grating, which was combined with an inverse Olympus microscope. The frequency doubled Nd:YAG laser (excitation wavelength $532 \mathrm{~nm}$ ) was focused into the micro cuvette with the help of an Olympus 50x UPlan FLN microscope objective. The incident laser power present on the sample was approximently $7 \mathrm{~mW}$. The $180^{\circ}$ back-scattered light was detected with back-illuminated CCD camera (1024 x 512 pixels).

For the SEM measurements silicon chips of $10 \mathrm{~mm} \times 10 \mathrm{~mm}$ were treated for $10 \mathrm{~min}$ in acetone and for $10 \mathrm{~min}$ in isopropanol in an ultrasonic bath. Afterwards the chips were washed with distilled water and dried using pressurized air. In order to investigate the EGNP modified magnetic beads $1 \mu \mathrm{l}$ of a sample was deposited on the silicon chips and dried. The images were acquired using a field emission scanning electron microscope (FESEM) JEOL JSM-6700F instrument (Tokyo, Japan). Additionally, SEM images in backscatter electron imaging mode (material contrast mode) were taken to obtain pictures with a higher contrast regarding their chemical composition.

The data evaluation was carried out using the open source script language GNU R [33]. The spectra were background corrected applying sensitive nonlinear iterative peak (SNIP) clipping algorithms before further evaluation.

\section{Results and Discussion}

A new approach for covering magnetic beads with a SERS active surface is investigated within this contribution. Enzymatically generated silver nanoparticles, which exhibit a nanoflower-like structure, are deposited at the surface of magnetic beads as described above and studied concerning the applicability for

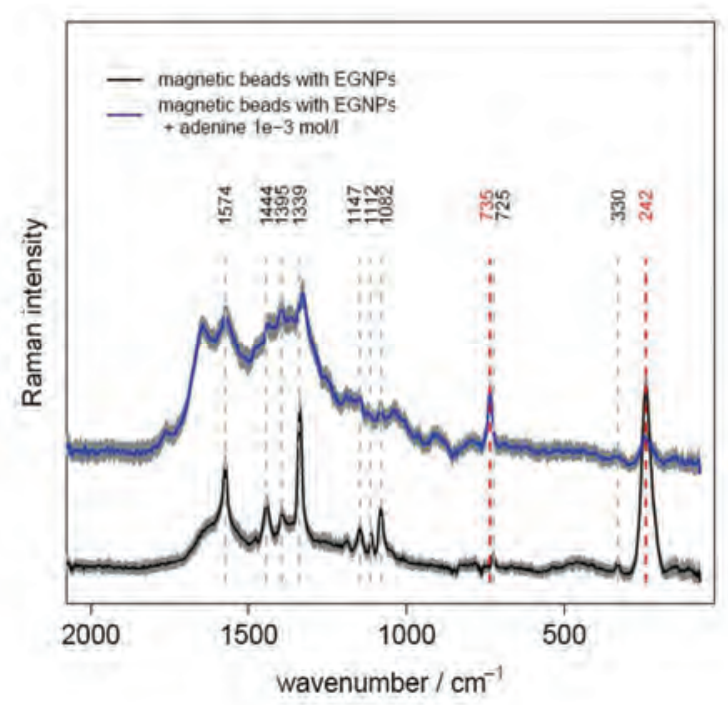

Fig. 3. SERS spectra of magnetic beads covered with EGNP with and without adenine $\left(c=1 \times 10^{-3} \mathrm{~mol} / \mathrm{l}\right)$

SERS measurements in solution. Figure 2 shows the SEM images (left side) and SEM images in material contrast mode (right side) of magnetic beads covered with EGNP. Applying SEM for characterization of the silver nanostructures on the magnetic beads, only morphology features such as small plates arranged like desert roses indicate the formation of the EGNP on the surface. Thus, images in backscatter electron imaging mode are recorded as well for the same image sections. Here, the silver structures appear in a high contrast with the magnetic beads due to the different chemical composition. It can be seen that the magnetic beads are covered with islands of nanoflowers.

Figure 3 shows a SERS spectrum of the magnetic beads covered with EGNP without an additional analyte (figure 3 black spectrum) as well as after the addition of an adenine solution with the concentration of $1 \times 10^{-3} \mathrm{~mol} / /$ (figure 3 blue spectrum). The pure suspension of EGNP covered magnetic bead shows a significant fingerprint SERS spectrum. According to literature the appearing bands can be attributed to the streptavidin-modified surface of the magnetic beads [34]. Galarreta et al. investigated streptavidin/biotin monolayer assemblies on gold substrates via SERS. In respect of the reported band assignment, the bands at 1574, 1444, 1339, 1147, 1112 and $1082 \mathrm{~cm}^{-1}$ can be related to streptavidin. The band at $242 \mathrm{~cm}^{-1}$ is typically assigned to a $v(\mathrm{Ag}$ $\mathrm{N})$ mode [35]. When adding a solution of adenine to EGNP covered magnetic beads the adenine ring breathing mode at $735 \mathrm{~cm}^{-1}$ can be observed in the SERS spectrum. The bands assigned to streptavidin decrease in intensity or are covered by background features. For the 


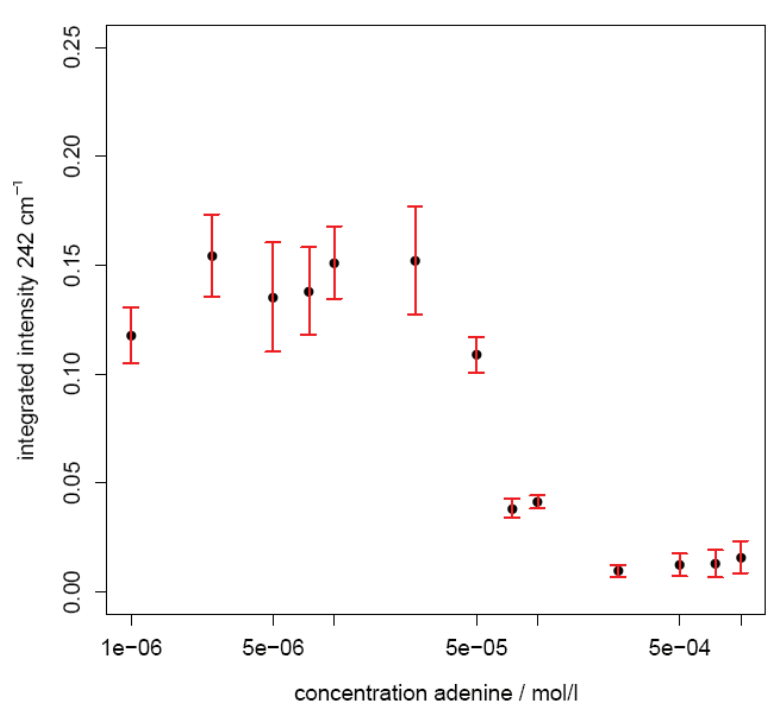

Fig. 4. Integrated Raman intensity of the band at $242 \mathrm{~cm}^{-1}$ within the SERS spectra at different adenine concentration

measurements with and without analyte the concentration of the magnetic beads with EGNP was identical. However, the intensity of the band at $242 \mathrm{~cm}^{-1}$ is drastically decreasing when adding adenine with a concentration of $1 \times 10^{-3} \mathrm{~mol} / \mathrm{l}$.

In order to investigate the change of the $\mathrm{v}(\mathrm{Ag}$ $\mathrm{N}$ ) mode in dependence of the adenine concentration, different concentrations of adenine were added to the magnetic beads. The detected SERS spectra were background corrected and normalized (I1 normalization) before further data evaluation. For all SERS spectra of the different adenine concentrations the $v(\mathrm{Ag}-\mathrm{N})$ mode at $242 \mathrm{~cm}^{-1}$ was integrated. In figure 4 the integrated Raman signal intensity of the band at $242 \mathrm{~cm}^{-1}$ is plotted as function of the adenine concentration. It should be noticed that the integrated Raman signal intensity stays constant up to a concentration of $2.5 \times 10^{-5} \mathrm{~mol} / \mathrm{l}$ adenine. With a concentration of $5 \times 10^{-5} \mathrm{~mol} / /$ adenine the integrated Raman intensity decreases. For concentrations from $2.5 \times 10^{-4}$ to $1 \times 10^{-3} \mathrm{~mol} / /$ the integrated signal intensity reaches a constant value again. It has to be mentioned that for this investigation the limit of detection for adenine is $1 \times 10^{-5} \mathrm{~mol} / \mathrm{l}$. For this concentration the adenine ring breathing mode is still observed. For lower concentration it does not appear within the spectra. The change of the integrated signal intensity of the band at $242 \mathrm{~cm}^{-1}$ results from the presence of adenine at the surface of the EGNP covered magnetic beads, but cannot explained in detail so far.

Investigating the SERS signal of the adenine ring breathing mode in dependence of the adenine concentration, the best correlation of

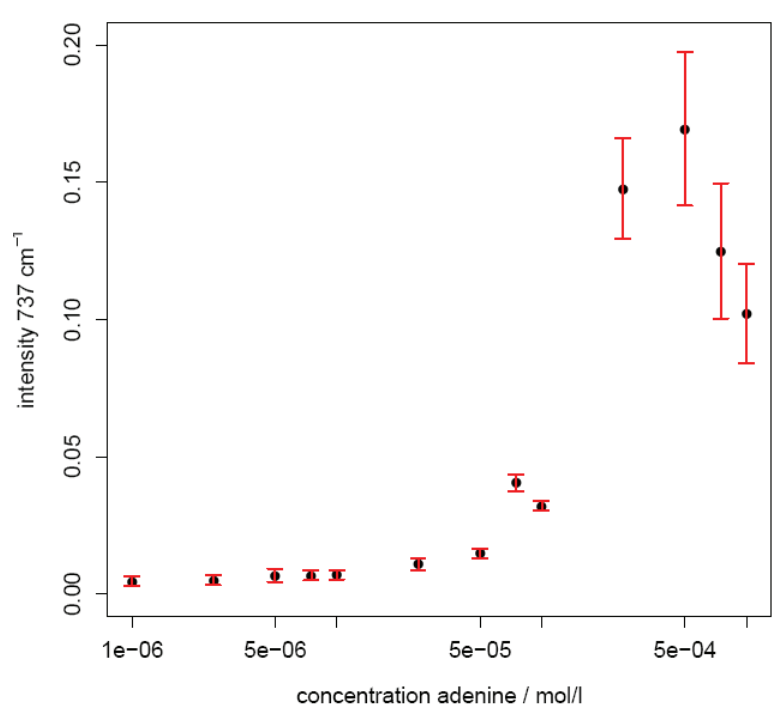

Fig. 5. Raman intensity of the adenine band at $737 \mathrm{~cm}^{-1}$ within the SERS spectra of different adenine concentration after normalization to the band at $242 \mathrm{~cm}^{-1}$

the signal intensity with the concentration can be found normalizing the background corrected SERS spectra to the respective integrated Raman signal intensity of the $\mathrm{v}(\mathrm{Ag}-\mathrm{N})$ mode at $242 \mathrm{~cm}^{-1}$ (figure 5). Here, a significant increase in respect to the background (concentration below $1 \times 10^{-5} \mathrm{~mol} / \mathrm{l}$, where no ring breathing mode of adenine appears at $735 \mathrm{~cm}^{-1}$ in the SERS spectra) is observed at a concentration of $2.5 \times 10^{-5} \mathrm{~mol} / \mathrm{l}$ adenine. The signal intensity for concentrations of adenine of $7.5 \times 10^{-4}$ to $1 \times 10^{-3} \mathrm{~mol} / /$ show an additional increase compare to the concentrations from $2.5 \times 10^{-5}$ to $5 \times 10^{-4} \mathrm{~mol} / \mathrm{l}$. This can be due to a complete coverage of the EGNP on the magnetic beads with adenine molecules. Below the concentration of $2.5 \times 10^{-4} \mathrm{~mol} / \mathrm{l}$ adenine the number of adenine molecules might not be sufficient to provide a continuous layer of molecules at the deposited EGNP on the magnetic beads. This would also explain that the signal intensity of the ring breathing mode drops with increasing concentration above $5 \times 10^{-5} \mathrm{~mol} / \mathrm{l}$ as a saturation of the existing silver surface at the magnetic beads is saturated with analyte molecules.

\section{Conclusion}

Within this contribution the high potential of magnetic beads covered with EGNP for the application in SERS diagnostics is demonstrated. However, the application of EGNP covered magnetic beads for SERS measurements still lacks of a sufficient sensitivity of the substrate. This is due to an inconsistent coverage of the surface of the magnetic beads with EGNP, which has to be 
improved and further investigated. However, this new approach for covering magnetic beads with a SERS active surface seems to be promising as it provides the following advantages: 1) the magnetic core allows a manipulation of the particles like trapping for detection or sample separation steps; 2) the EGNP provide a unique nanoflower structure well suited for SERS detection; 3) EGNP can be produced easily and cost efficient.

\section{Acknowledgement}

We thank Franka Jahn for measuring the SEM images. Funding of the research projects 'QuantiSERS' and 'Jenaer Biochip Initiative 2.0' within the framework 'Unternehmen Region: InnoProfile-Transfer' from the Federal Ministry of Education and Research, Germany (BMBF) is gratefully acknowledged.

\section{References}

[1] A. Campion, P. Kambhampati, Surface-enhanced Raman scattering, Chemical Society Reviews 27, 241-250 (1998); doi: 10.1039/A827241Z

[2] M. Kerker, D.S. Wang, H. Chew, Surface enhanced Raman scattering (SERS) by molecules adsorbed at spherical particles Applied Optics 19, 3373-3388 (1980); doi: 10.1364/AO.19.003373

[3] M. Moskovits, Surface-enhanced spectroscopy, Reviews of Modern Physics 57, 783-826 (1985); doi: 10.1103/RevModPhys.57.783

[4] D. Cialla, A. Maerz, R. Boehme, F. Theil, K. Weber, M. Schmitt, J. Popp, Surface-enhanced Raman spectroscopy (SERS): progress and trends, Analytical and Bioanalytical Chemistry 403, 27-54 (2012); doi: 10.1007/s00216-011$5631-x$

[5] R. Das, R. Jagannathan, C. Sharan, U. Kumar, P. Poddar, Mechanistic Study of Surface Functionalization of Enzyme Lysozyme Synthesized Ag and Au Nanoparticles Using Surface Enhanced Raman Spectroscopy, Journal Of Physical Chemistry C 113, 21493-21500 (2009); doi: 10.1021/jp905806t

[6] L. Rivas, S. Sanchez-Cortes, J.V. Garcia-Ramos, G. Morcillo, Mixed silver/gold colloids: A study of their formation, morphology, and surfaceenhanced Raman activity, Langmuir 16, 97229728 (2000); doi: 10.1021/la000557s

[7] I.W. Sztainbuch, The effects of Au aggregate morphology on surface-enhanced Raman scattering enhancement, Journal of Chemical Physics 125, 124707 (2006); doi: 10.1063/1.2338029

[8] D. Cialla, U. Huebner, H. Schneidewind, R. Moeller, J. Popp, Probing innovative microfabricated substrates for their reproducible SERS activity, ChemPhysChem 9, 758-762 (2008); doi: 10.1002/cphc.200700705
[9] U. Huebner, R. Boucher, H. Schneidewind, D. Cialla, J. Popp, Microfabricated SERS-arrays with sharp-edged metallic nanostructures, Microelectronic Engineering 85, 1792-1794 (2008); doi: 10.1016/j.mee.2008.05.005

[10] M. Baia, L. Baia, S. Astilean, J. Popp, Surfaceenhanced Raman scattering efficiency of truncated tetrahedral Ag nanoparticle arrays mediated by electromagnetic couplings, Applied Physics Letters 88, 143121 (2006); doi: 10.1063/1.2193778

[11] A.J. Haes, C.L. Haynes, A.D. McFarland, G.C. Schatz, R.R. Van Duyne, S.L. Zou, Plasmonic materials for surface-enhanced sensing and spectroscopy, MRS Bulletin 30, 368-375 (2005); doi: $10.1557 / \mathrm{mrs} 2005.100$

[12] X. Hou, Y. Fang, Surface-enhanced Raman scattering of single-walled carbon nanotubes on modified silver electrode, Spectrochimica Acta Part A-Molecular And Biomolecular Spectroscopy 69, 1140-1145 (2008); doi: 10.1016/j.saa.2007.06.033

[13] D. Li, D.-W. Li, J.S. Fossey, Y.-T. Long, In situ surface-enhanced Raman scattering and X-ray photoelectron spectroscopic investigation of coenzyme Q(10) on silver electrode, Physical Chemistry Chemical Physics 13, 2259-2265 (2011); doi: 10.1039/C0CP01449D

[14] K.K. Strelau, T. Schueler, R. Moeller, W. Fritzsche, J. Popp, Novel Bottom-Up SERS Substrates for Quantitative and Parallelized Analytics, ChemPhysChem 11, 394-398 (2010); doi: 10.1002/cphc.200900867

[15] K.K. Strelau, A. Brinker, C. Schnee, K. Weber, R. Moeller, J. Popp, Detection of PCR products amplified from DNA of epizootic pathogens using magnetic nanoparticles and SERS, Journal of Raman Spectroscopy 42, 243-250 (2011); doi: $10.1002 / j r s .2730$

[16] J. Kneipp, H. Kneipp, K. Kneipp, SERS--a singlemolecule and nanoscale tool for bioanalytics, Chemical Society Reviews 37, 1052-1060 (2008); doi: 10.1039/B708459P

[17] A. März, B. Mönch, P. Rösch, M. Kiehntopf, T. Henkel, J. Popp, Detection of thiopurine methyltransferase activity in lysed red blood cells by means of lab-on-a-chip surface enhanced Raman spectroscopy (LOC-SERS), Analytical and Bioanalytical Chemistry 400, 2755-2761 (2011); doi: 10.1007/s00216-011-4811-z

[18] S. Pahlow, A. Maerz, B. Seise, K. Hartmann, I. Freitag, E. Kaemmer, R. Boehme, V. Deckert, K. Weber, D. Cialla, J. Popp, Bioanalytical application of surface- and tip-enhanced Raman spectroscopy, Engineering In Life Sciences 12, 131-143 (2012); doi: 10.1002/elsc.201100056

[19] X.M. Qian, S.M. Nie, Single-molecule and singlenanoparticle SERS: from fundamental mechanisms to biomedical applications, Chemical Society Reviews 37, 912-920 (2008); doi: 10.1039/B708839F 
[20] M. Salehi, D. Steinigeweg, P. Ströbel, A. Marx, J. Packeisen, S. Schlücker, Rapid immuno-SERS microscopy for tissue imaging with singlenanoparticle sensitivity, Journal of Biophotonics 6, 785-792 (2013); doi: 10.1002/jbio.201200148

[21] P. Negri, R.A. Dluhy, Ag nanorod based surfaceenhanced Raman spectroscopy applied to bioanalytical sensing, Journal of Biophotonics 6 , 20-35 (2013); doi: 10.1002/jbio.201200133

[22] U.S. Dinish, G. Balasundaram, Y.T. Chang, M. Olivo, Sensitive multiplex detection of serological liver cancer biomarkers using SERS-active photonic crystal fiber probe, Journal of Biophotonics (published online); doi: 10.1002/jbio.201300084

[23] C. Yuen, Q. Liu, Towards in vivo intradermal surface enhanced Raman scattering (SERS) measurements: silver coated microneedle based SERS probe, Journal of Biophotonics (published online); doi: 10.1002/jbio.201300006

[24] F. Bao, J.-L. Yao, R.-A. Gu, Synthesis of Magnetic $\mathrm{Fe}_{2} \mathrm{O}_{3} / \mathrm{Au}$ Core/Shell Nanoparticles for Bioseparation and Immunoassay Based on Surface-Enhanced Raman Spectroscopy, Langmuir 25, 10782-10787 (2009); doi: 10.1021/la901337r

[25] M. Fan, G.F.S. Andrade, A.G. Brolo, A review on the fabrication of substrates for surface enhanced Raman spectroscopy and their applications in analytical chemistry, Analytica Chimica Acta 693, 7-25 (2011); doi: 10.1016/j.aca.2011.03.002

[26] B. Han, N. Choi, K.H. Kim, D.W. Lim, J. Choo, Application of Silver-Coated Magnetic Microspheres to a SERS-Based Optofluidic Sensor, Journal of Physical Chemistry C 115, 6290-6296 (2011); doi: 10.1021/jp112265e

[27] K. Kim, J.-Y. Choi, H.B. Lee, K.S. Shin, Silanization of Ag-Deposited Magnetite Particles: An Efficient Route to Fabricate Magnetic Nanoparticle-Based Raman Barcode Materials, ACS Applied Materials \& Interfaces 2, 1872-1878 (2010); doi: 10.1021/am1002074

[28] K. Kim, H.J. Jang, K.S. Shin, Ag nanostructures assembled on magnetic particles for ready SERS-based detection of dissolved chemical species, Analyst 134, 308-313 (2009); doi: 10.1039/B811425K

[29] P.A. Mosier-Boss, S.H. Lieberman, Surfaceenhanced Raman spectroscopy substrate composed of chemically modified gold colloid particles immobilized on magnetic microparticles, Analytical Chemistry 77, 1031-1037 (2005); doi: 10.1021/ac048647f

[30] Y. Wang, S. Ravindranath, J. Irudayaraj, Separation and detection of multiple pathogens in a food matrix by magnetic SERS nanoprobes, Analytical and Bioanalytical Chemistry 399, 1271 1278 (2011); doi: 10.1007/s00216-010-4453-6

[31] J. Du, C. Jing, Preparation of Thiol Modified $\mathrm{Fe}_{3} \mathrm{O}_{4} @ \mathrm{Ag}$ Magnetic SERS Probe for PAHs Detection and Identification, Journal Of Physical
Chemistry C 115, 17829-17835 (2011); doi: 10.1021/jp203181c

[32] B.-H. Jun, M.S. Noh, J. Kim, G. Kim, H. Kang, M.S. Kim, Y.-T. Seo, J. Baek, J.-H. Kim, J. Park, S. Kim, Y.-K. Kim, T. Hyeon, M.-H. Cho, D.H. Jeong, Y.-S. Lee, Multifunctional Silver-Embedded Magnetic Nanoparticles as SERS Nanoprobes and Their Applications, Small 6, 119-125 (2010); doi: 10.1002/smll.200901459

[33] R Development Core Team, R: A Language and Environment for Statistical Computing, Vienna, Austria (2011); ISBN 3-900051-07-0

[34] B.C. Galarreta, P.R. Norton, F. LagugneLabarthet, SERS Detection of Streptavidin/Biotin Monolayer Assemblies, Langmuir 27, 1494-1498 (2011); doi: 10.1021/la1047497

[35] A. Vivoni, R.L. Birke, R. Foucault, J.R. Lombardi, $A b$ initio frequency calculations of pyridine adsorbed on an adatom model of a SERS active site of a silver surface, Journal of Physical Chemistry B 107, 5547-5557 (2003); doi: 10.1021/jp027642o 